\title{
A review of capecitabine-based adjuvant therapy for gastric cancer in the Chinese population
}

\author{
Xiangdong Cheng*,1 \& Yi Lu \\ ${ }^{1}$ Department of Gastrointestinal Surgery, Zhejiang Provincial Hospital of TCM, Hangzhou, Zhejiang, PR China \\ ${ }^{2}$ Medical Department, Shanghai Roche Pharmaceutical Company, Shanghai, PR China \\ * Author for correspondence: Tel.: +86571 85827888; Fax: +86571 85827511; chengxd516@126.com
}

In China, the treatment of locally advanced gastric cancer (AGC) faces unique challenges. Chinese patients may harbor more unfavorable prognostic factors than western populations and, in comparison with other Asian populations such as Japan and South Korea, a higher proportion of Chinese patients are diagnosed with AGC due to inadequate early diagnosis of the malignancy. This review summarizes the use of combination chemotherapy regimens with capecitabine as adjuvant therapy in the Chinese AGC population. Based on the available domestic data in China, the review concludes that capecitabine-based chemotherapy regimens, especially XELOX, offer good efficacy following radical gastrectomy in patients with AGC, with a low incidence of adverse events, acceptable tolerance, greater patient convenience and a lower overall cost than other regimens.

First draft submitted: 20 October 2017; Accepted for publication: 24 November 2017; Published online: 18 December 2017

Keywords: adjuvant therapy $\bullet$ capecitabine $\bullet$ Chinese population $\bullet$ D2 dissection $\bullet$ gastric cancer

Gastric cancer (GC) is the fifth most-common malignancy and the third leading cause of cancer-related deaths worldwide, with the highest mortality rates reported in East Asia [1]. In China, GC is the second most frequently diagnosed cancer, with an estimated 679,100 new cases reported in 2015, accounting for $>40 \%$ of the worldwide annual incidence [2]. Although the 5-year survival rate has been increasing in last two decades [3], GC remains a major cause of death in China, with an estimated 498,000 new deaths occurring in 2015 [2].

Surgery is still the optimal curative treatment for GC. However, the majority of patients are found to be suffering from locally advanced GC (AGC) on initial diagnosis, which leads to a high risk of recurrence and poor longterm outcomes [4]. A series of research efforts has been undertaken to improve the postoperative survival of AGC patients. First, historical data and recent studies have shown that D2 lymph node dissection (extended resection) with spleen and pancreas preservation leads to significantly better outcomes than D1 dissection [5,6]. Second, several Phase III randomized, controlled trials (RCTs), including the CLASSIC, INT0116, MAGIC, FNLCC/FFCD and ACT-GC studies, have demonstrated significant survival advantages with perioperative or adjuvant chemotherapy in AGC patients [4,7]. Adjuvant chemotherapy, notably with the XELOX regimen (capecitabine plus oxaliplatin), can significantly improve the long-term survival and reduce the risk of death of patients postoperatively, regardless of ethnic and regional differences. In the CLASSIC study, XELOX chemotherapy following D2 gastrectomy in patients with AGC led to an obvious improvement in survival compared with surgery alone (5-year disease-free survival [DFS]: 67 vs 53\%, respectively; $\mathrm{p}<0.0001)$ and a 34\% reduction in the risk of death $(\mathrm{p}=0.0015)$ [8]. Therefore, authoritative consensus and practice guidelines for the USA (National Comprehensive Cancer Network guidelines) [9], Europe (European Society for Medical Oncology guidelines) [5] and Asia [10] have recommended adjuvant chemotherapy (e.g., XELOX) combined with radical surgery as one of the standard treatment options for $\geq \mathrm{T} 2$ or $\mathrm{N}^{+}$resectable AGC patients.

In China, the treatment of AGC treatment faces unique challenges. In comparison with western populations, the Chinese population may harbor more unfavorable factors associated with poor survival, such as later-stage disease, larger tumor sizes and a higher incidence of positive lymph nodes [4]. In addition, in comparison with other Asian 
populations, a higher proportion of patients are diagnosed with AGC due to inadequate early diagnosis (80\% in China vs $<50 \%$ in Japan and South Korea [7]) and this has become another dilemma for clinical management of Chinese patients. Even with more aggressive D2 dissection surgery and adoption of the adjuvant treatments utilized in other Asian countries, the prognosis for patients with AGC in China is still significantly worse than that in Japan and South Korea (5-year survival rates: 30 vs $\geq 50 \%$ ) [3]. Therefore, instituting the treatments adopted in Japan and South Korea has not proved feasible for Chinese clinicians, and a large number of studies of adjuvant chemotherapy for AGC have now been conducted in China to determine the optimal strategy. As with the results of studies conducted in other countries, XELOX chemotherapy has significantly improved the survival of Chinese postoperative AGC patients, and this has become a commonly used regimen in adjuvant settings in China [11]. This review summarizes the use of combination chemotherapy regimens (e.g., XELOX) and other studies with capecitabine as adjuvant therapy to explore adjuvant chemotherapy models for the Chinese AGC population. Due to limited data in China, we focused on capecitabine-based adjuvant therapy for GC in the Chinese population, though not specifically on D2 dissection.

A literature search of PubMed, Google Scholar and the Chinese Wanfang and China National Knowledge Infrastructure databases were conducted up to 11 June 2017. The search terms used were: 'capecitabine', 'advanced gastric cancer' and 'Chinese population' or 'China'. Articles referring to adjuvant or postoperative therapy were included while those referring to subsequent therapy were excluded. Due to the limited numbers of studies and the relatively small sample sizes of studies conducted in China, different types of research studies, including randomized, retrospective and observational studies, were included to collect as much data as possible.

\section{Capecitabine-based chemotherapy}

Various capecitabine-based chemotherapy regimens have been used in the treatment of GC. XELOX, which comprises oral capecitabine $\left(1000 \mathrm{mg} / \mathrm{m}^{2}\right.$ b.i.d. on days $1-14$, once every 3 weeks [q3w]) and intravenous oxaliplatin $\left(130 \mathrm{mg} / \mathrm{m}^{2} \mathrm{qd}\right.$ on day $\left.1, \mathrm{q} 3 \mathrm{w}\right)$, is one of the most commonly used regimens. A series of clinical studies have been conducted with XELOX in Chinese patients with AGC.

The CLASSIC study was an open-label, parallel-group, multiregion (mainland China, Taiwan and South Korea), Phase III RCT that compared surgery with and without XELOX adjuvant chemotherapy in stage II-IIIB GC patients who had undergone D2 dissection [8,11]. Analysis of the Chinese subgroup $(\mathrm{n}=100)$ showed a significantly higher 3-year DFS rate (78 vs 56\%, HR: 0.56; $\mathrm{p}<0.0001$ ) and a reduced risk of relapse ( $59 \%$ reduction, $\mathrm{p}=0.013$ ) in the surgery + XELOX group than in the surgery-only group; however, the 3-year overall survival (OS) rate was similar in the two arms (78 vs 66\%; $\mathrm{p}=0.110)$. Grade III or IV adverse events (AEs) in the surgery + XELOX group included neutropenia $(63.5 \%)$, thrombocytopenia $(36.5 \%)$, vomiting $(44.2 \%)$, decreased appetite $(34.6 \%)$ and loss of bodyweight (23.1\%) [8]. Similar with results were recorded in the overall population of the CLASSIC study, indicating that adjuvant XELOX chemotherapy improved outcomes in patients with AGC after D2 gastrectomy and had an acceptable safety profile [8].

Three studies compared adjuvant chemotherapy with XELOX with the FOLFOX regimen (oxaliplatin, 5fluorouracil [5-FU] and folinic acid) in patients with AGC who underwent D2 dissection [12-14]. Two of these studies $[12,14]$ found that XELOX significantly improved the 1-year DFS $(\mathrm{p}<0.05)$ and progression-free survival (PFS; $\mathrm{p}<0.05$ ) to a greater degree than FOLFOX, but the third study [13] showed that XELOX did not offer a greater survival benefit than FOLFOX. Several retrospective studies have also reported that the efficacy of XELOX was similar to that of FOLFOX [15-17]. However, it is worth noting that the incidence of AEs was lower in patients receiving XELOX than in those receiving FOLFOX, and more patients were able to complete XELOX chemotherapy than FOLFOX chemotherapy [13]. This finding implies that capecitabine has better tolerance than 5-FU + folinic acid.

Two studies (one RCT and one retrospective study) have reported that the efficacy of XELOX as adjuvant chemotherapy in patients with AGC was comparable to that of the SOX regimen (S-1 combined with oxaliplatin) [18]. Furthermore, Li et al. [19] compared the efficacy and safety of XELOX with that of XELLOBA (capecitabine + lobaplatin) after D2 resection. Both the 3-year DFS with the XELOX and XELLOBA regimens ( 62.9 vs $75.0 \%$, respectively; $\mathrm{p}=0.253$ ) and the incidence of AEs ( 20.0 vs $21.42 \%$, respectively; $\mathrm{p}=0.889$ ) were not significantly different between the two adjuvant therapy groups [19]. 


\section{Capecitabine-based concurrent chemoradiotherapy}

Postoperative chemoradiotherapy (CRT) is currently a standard treatment option in the USA for patients with resected GC based on the results of the Intergroup 0116 (INT0116) study [20]. Another Phase III multicenter study (CRITICS) aimed to assess whether the combination of preoperative chemotherapy and postoperative CRT can improve the clinical outcome compared with the current European standard of perioperative chemotherapy [21]. It is well accepted that postoperative regional radiation plus chemotherapy reduces the risk of relapse and prolongs survival in high-risk AGC patients following D0 or D1 dissection. However, whether postoperative adjuvant CRT will benefit patients after D2 dissection has been a matter of debate in Japan and South Korea for some time, and it is also unclear in China.

As a radiosensitizer, oral capecitabine is considered to be a promising option in both pre- and post-operative CRT, encouraging the performance of several capecitabine-related CRT studies [22,23]. An RCT by Shi et al. [24] in 110 Chinese AGC patients showed a significantly improved survival with CRT (XELOX + 3D conformal radiotherapy $[\mathrm{RT}]$ ) versus chemotherapy (XELOX) alone. Compared with XELOX alone, XELOX plus RT significantly improved the 3-year OS (58.18\% in the CRT arm vs 32.73\% in the XELOX alone arm; $\mathrm{p}<0.05)$, and reduced both the 3 -year locoregional recurrence rate (3.64 vs $23.64 \%$, respectively; $\mathrm{p}<0.05)$ and the distant metastasis rate $(14.55$ vs $49.09 \%$, respectively; $\mathrm{p}<0.05)$. However, there was no statistically significant difference in the incidence of grade III-IV AEs between the two arms of the study. The 3-year OS in this trial was lower than in the CLASSIC study [11] (32.73 vs 78\%, respectively), which may be explained by the different inclusion criteria of the two studies. Whereas Shi et al. recruited patients with TNM stages T3-4 AGC, the CLASSIC study recruited patients with stages II-III AGC. Similar findings to those of Shi et al. [24] were reported in three small-scale RCTs (Chen et al. [capecitabine + RT vs capecitabine alone] [25], Guo et al. [docetaxel + capecitabine + RT vs chemotherapy alone] [26] and Zhang et al. [27]). A significantly improved 5-year OS (61.1\% in the CRT arm vs $38.9 \%$ in the chemotherapy arm; $\mathrm{p}<0.05)$ and decreased locoregional recurrence rate (19.4 vs $70.3 \%$, respectively; $\mathrm{p}<0.05$ ) were reported by Chen et al. [25], while Guo et al. [26] found that the 3-year OS was improved in the CRT arm in comparison with chemotherapy alone (55.26 vs $28.95 \%$, respectively). Zhang et al. [27] also demonstrated a survival benefit in the 3-year relapse-free survival rate with the combination of capecitabine-based chemotherapy and RT compared with chemotherapy alone ( 48.24 vs $28.52 \%$, respectively; $\mathrm{p}<0.05$ ).

In a retrospective study that compared XELOX-based CRT and XELOX alone in 79 patients with recurrent locoregional GC after curative dissection [28], a significantly enhanced overall response (complete response [CR] plus partial response [PR]: 87.8 vs $63.2 \%$, respectively; $\mathrm{p}=0.01$ ) and a prolonged median OS (13.4 vs 5.4 months, respectively; $\mathrm{p}=0.06$ ) were found in the CRT arm (involved-field RT + XELOX) compared with the XELOXalone arm; however, there was no significant difference in the incidence of AEs between the two arms [28]. Luo et al. [29] evaluated the clinical impact of intensity-modulated radiation therapy combined with different concurrent chemotherapies (including intravenous infusion of 5-FU [F-CRT] or oral capecitabine [C-CRT]) after D2 dissection in patients with GC [29]. Both the F-CRT and C-CRT arms were found to be effective (3-year OS: $72.2 \%$ in the F-CRT arm vs $78.5 \%$ in the C-CRT arm; $\mathrm{p}>0.05$ ) and well tolerated. These results were in accord with studies performed by Ren et al. [30] and Wang et al. [31]. In terms of influence on quality-of-life, Qiu et al. [23] found that capecitabine was superior to 5-FU and worthy of being regarded as a standard treatment for CRT.

Capecitabine-based CRT was well tolerated in these studies. The incidence of grade III or IV hematological toxicity ranged from 8.3 to $24.4 \%$ in five evaluable trials (total number of patients 221 [29-33], while that of gastrointestinal toxicity (including nausea, vomiting and diarrhea) ranged from 8.1 to $42 \%$ in seven evaluable trials (total number of patients 338) [23,27,29-33], and grade III-IV hand-foot syndrome (HFS) ranged from 1.8 to $2.7 \%$ in two evaluable trials [23,29].

Comparisons of the efficacy of adjuvant CRT in different regions of the world have not proved feasible as surgical principles differ between eastern and western countries [34]. Although postoperative CRT after D0 or D1 dissection (a standard option in Europe and the USA) showed significant survival benefit [20,21], postoperative CRT after D2 dissection (a standard option in east Asia) did not contribute to any significant reduction in the risk of recurrence in the ARTIST trial in South-Korean patients [35]. While most patients in the INT0116 study [20] had T3/T4 disease and $85 \%$ had nodal metastases, almost $60 \%$ of patients had early stage disease (IB/IIA) in the ARTIST trial [35]. Since extended D2 dissection and standard adjuvant chemotherapy offer adequate survival benefit for early stage GC, treatment with CRT seemed an unnecessary option for patients in the ARTIST trial. Therefore, adjuvant CRT 
may be best considered an option in high-risk patients with node-positive disease (T3, T4) or following suboptimal surgery (less than D2) [36].

In brief, capecitabine-based CRT following radical surgery for GC has proved safe and effective in Chinese patients. However, more evidence is needed to confirm its applicability.

\section{Capecitabine monotherapy}

Although only limited data are available on the efficacy and safety of capecitabine monotherapy in China, several studies have suggested that it may be a suitable option for elderly patients with AGC [37-40]. In an RCT [38], 60 elderly stage II-III GC patients who had undergone dissection were randomly assigned to either high-dose capecitabine $\left(2500 \mathrm{mg} / \mathrm{m}^{2}\right.$ b.i.d. on days $1-14$, q3w) or low-dose capecitabine $\left(2000 \mathrm{mg} / \mathrm{m}^{2}\right.$ b.i.d. on days $1-14$, q3w). After six treatment cycles, there was no statistically significant difference between the two groups in 2-year survival rate ( 80 vs $77 \%$, respectively; $\mathrm{p}=0.45$ ) and relapse-free survival ( 19.2 vs 19.0 months, respectively; $\mathrm{p}=0.36$ ). However, there was a higher incidence of AEs in the high-dose group. For example, more patients in the high-dose group suffered from grade III-IV AEs such as oral mucositis (6.67 vs $0.00 \%$, respectively), hyperbilirubinemia (6.67 vs $3.33 \%)$ and HFS (16.67 vs 6.67\%) than those in the low-dose group [38].

In a retrospective study conducted in China [37] that evaluated 38 elderly AGC patients treated with capecitabine $\left(2500 \mathrm{mg} / \mathrm{m}^{2}\right.$ b.i.d. on days $1-14, \mathrm{q} 3 \mathrm{w}$ ) after gastrectomy, the objective response rate was $47.37 \%$ (three patients had a CR and 15 had a PR). The most common AEs were HFS (grade III in 5.26\% of patients), which was able to be relieved after drug withdrawal or symptomatic treatment. Thus, capecitabine monotherapy has proved safe and effective in elderly Chinese patients with AGC.

\section{Capecitabine combined with intraperitoneal chemotherapy}

Intraperitoneal administration of cytotoxic agents combined with systemic chemotherapy has been attempted in patients after D2 dissection to prevent peritoneal metastasis and enhance antitumor efficacy. Intraperitoneal chemotherapy (IPC) allows drugs to achieve a high intraperitoneal concentration and to directly act on free tumor cells and peritoneal nodes. Drugs absorbed through the peritoneum enter the portal vein and also have a chemotherapeutic action on liver metastases [40]. A meta-analysis of 20 prospective RCTs has shown that IPC has a positive effect in preventing peritoneal recurrences and distant metastasis [41].

An RCT that randomly assigned 80 patients to either adjuvant chemotherapy with intraperitoneal cisplatin plus oral capecitabine or intravenous cisplatin plus oral capecitabine after dissection [42] found that 2-year peritoneal and liver metastasis rates were significantly lower in the IPC group than in intravenous chemotherapy group (peritoneal metastasis rate: 42.5 vs $72.5 \%$, respectively; $\mathrm{p}<0.01$; liver metastasis rate: 15.0 vs $37.5 \%$, respectively; $\mathrm{p}<0.05$ ). In addition, the occurrence of AEs was significantly lower in the IPC group (myelosuppression: 57.5 vs $80.5 \%$, respectively; $\mathrm{p}<0.05$; gastrointestinal AEs: 52.5 vs $85.0 \%$, respectively; $\mathrm{p}<0.01$ ) [42].

An observational study that enrolled 20 GC patients with pyoperitoneum [43] found that after two cycles of intraperitoneal docetaxel and cisplatin plus oral capecitabine treatment, $60 \%$ of patients achieved an objective response (25\% had a CR and 35\% had a PR). No grade III-IV AEs were reported in this study [43].

\section{Capecitabine maintenance therapy}

Several Chinese studies have reported the efficacy and safety of the continuous maintenance therapy with capecitabine in AGC patients. Feng et al. [44] found that patients benefited from prolonged adjuvant capecitabine therapy. In a study in which 307 GC patients who had undergone D2 dissection were randomized to XELOX or XELOX plus prolonged capecitabine chemotherapy $\left(1000 \mathrm{mg} / \mathrm{m}^{2}\right.$ b.i.d. on days $1-14, \mathrm{q} 3 \mathrm{w}$ for an additional eight cycles after eight cycles of XELOX), the 3-year DFS was significantly improved in the prolonged chemotherapy group (56.6 vs $48.4 \%$ in the XELOX group; $\mathrm{p}=0.0357$ ). The benefit of capecitabine maintenance therapy was most remarkable in patients with stage IIIA GC, in whom there was a significantly higher DFS (50.0 vs 40.96\%, respectively; $\mathrm{p}=0.0178)$ and OS (71.95 vs $57.83 \%$, respectively; $\mathrm{p}=0.023)$ in comparison with the XELOX group. Although more participants in the prolonged chemotherapy group experienced HFS, no grade IV AEs or treatment-related deaths were reported.

In another observational study in 26 GC patients who received R0 resection (D1, $\mathrm{n}=10 ; \mathrm{D} 2, \mathrm{n}=16)$ and six cycles of adjuvant chemotherapy [45], the time to progression in 18 evaluable patients was 14.7 months and the 1-year recurrence rate was $23.1 \%$ [45]. Grade III-IV AEs included myelosuppression (7.7\% of patients), HFS 
(3.8\%) and diarrhea (3.8\%). In general, the efficacy and safety of capecitabine maintenance therapy in Chinese patients with AGC have been promising and point to a need for further exploratory studies.

\section{Applicability of oral capecitabine \& pharmacoeconomic considerations}

The availability of oral fluoropyrimidine therapy has provided dosage schedule flexibility and avoided common problems associated with intravenous 5-FU therapy. Oral capecitabine treatment has been found to provide comparative efficacy with reduced toxicity, improved patient acceptance and more convenience than intravenous 5-FU in gastric and colorectal cancers [46-49]. Therefore, most patients are inclined to choose oral rather than intravenous therapy.

Pharmacoeconomic analysis in Europe and the USA has shown that overall costs can be significantly reduced with the use of capecitabine instead of 5-FU + folinic acid [50,51]. In view of its pharmacoeconomic benefits, the public health departments of several countries including the USA (US FDA), UK (NHS) and China (CFDA) have recommended that the costs of capecitabine treatment for malignancies such as advanced breast cancer and colorectal cancer should be covered by medical insurance schemes [50,51]. The efficacy and convenience of capecitabine as adjuvant therapy for postoperative AGC patients has also been recognized in China. In a pharmacoeconomic analysis of adjuvant therapies in patients with stage II-III GC who had undergone D2 dissection, Wu et al. [52] found that XELOX induced a greater increase in quality-adjusted life years (QALYs) over the course of the disease than either S-1 treatment or gastrectomy alone (8.1 QALYs as compared with 7.8 QALYs with S-1 and 6.2 QALYs with gastrectomy alone).

\section{Discussion}

As a well-accepted oral fluoropyrimidine drug internationally, capecitabine-containing regimens such as XELOX have become the recommended standard adjuvant treatment following radical gastrectomy for AGC patients in many western and east-Asian countries [5,9,10], mainly based on the survival benefit reported in the CLASSIC trial [8] in stage II-IIIB GC patients who received XELOX following D2 dissection. Capecitabine-based adjuvant regimens (especially XELOX) have also been commonly used in China, but the optimal regimen and the clinical decisionmaking steps cannot easily be duplicated from other countries because GC in China has unique characteristics, including later-stage disease, larger tumor sizes and a higher incidence of positive lymph nodes in comparison with western communities [4], and the disease status in the majority of patients differs from that in other eastAsian countries (advanced-stage disease in China vs early stage disease in Japan and Korea) [7]. Moreover, surgical management differs from western countries in that D2 dissection is preferred in China, whereas D0 and D1 dissection are preferred in Europe and the USA [34]. Therefore, a series of investigations has been conducted to determine the optimal Chinese-specific adjuvant regimens for AGC patients.

Consistent with studies in other countries, capecitabine-based adjuvant regimens have been found to offer a significant survival benefit and a tolerable safety profile for postoperative AGC patients in China [11]. In patients receiving capecitabine-containing adjuvant chemotherapy (XELOX), CRT, or capecitabine maintenance therapy following D2 dissection, the 3-year DFS in various studies has been reported to be $67.2-78 \%$ (with a similar 5-year DFS) [11], 55.3-72.2\% [29-36] and 57\% [49,50]. A comparison of DFS rates among different capecitabine-based regimens has not proved feasible as the baseline characteristics of AGC patients in the various studies are often different, and patients who receive CRT or maintenance capecitabine therapy often have a higher risk of relapse.

As the most commonly used regimen, several studies have compared XELOX with other antitumor regimens. Compared with FOLFOX [14-19], XELOX has shown better or similar efficacy and a lower incidence of toxicity, and compared with the XELLOBA and SOX regimens $[20,21]$ it has shown equivalent efficacy and a similar safety profile. It is notable that while no survival benefit from S- 1 was observed in elderly AGC patients $\geq 70$ years of age [53], capecitabine had promising survival benefits (2-year OS: 77-80\%) and favorable tolerance [41-44] and may be a good choice for this subgroup of patients.

Certain treatment options may achieve better patient outcomes under specific conditions. XELOX, which has been the most commonly used adjuvant regimen, is suitable for the general AGC population. Capecitabine-based CRT, which has stronger antitumor activity but more severe toxicity, may be a suitable option for high-risk patients with later-stage or node-positive disease (T3, T4) or where suboptimal surgery (less than D2) has been performed, while low-dose capecitabine monotherapy may be more feasible for elderly patients with AGC. Capecitabine maintenance therapy, which has proved well tolerated and efficient in reducing recurrences, might be an option for patients with a high risk of relapse, and intraperitoneal regimens, which have had a positive effect in decreasing ascitic 
fluid and preventing peritoneal recurrence and distant metastasis (especially liver metastasis), can be considered for patients with pyoperitoneum, ascites and a high risk of metastasis [54].

Overall, capecitabine-based adjuvant therapy has been found to improve the postoperative survival rate and reduce recurrences and metastasis (especially liver metastasis [54]) in Chinese patients with AGC. When patient tolerance, treatment completion rates and quality-of-life are taken into consideration, capecitabine-based adjuvant therapy appears to be a suitable choice for AGC patients.

There are some limitations to the findings summarized in this analysis. First, the limited number of Chinese studies and relatively small sample sizes may have affected the statistical conclusions. Second, only some of the studies analyzed were RCTs, and even in these studies, the methods adopted for randomization and blindness were not strictly standard. Third, missing follow-up data have been a common deficiency of Chinese studies, leading to a lack of long-term data and grade 1 evidence. Therefore, further large-scale, prospective, multicenter Phase III studies in both the general population and specific subgroups of AGC patients should be conducted to determine the optimal postoperative strategy.

\section{Conclusion}

On the basis of domestic clinical data in China, capecitabine-based chemotherapy regimens, especially XELOX, appear to offer good efficacy following radical gastrectomy in Chinese patients with AGC, with a low incidence and severity of AEs, acceptable tolerance, greater patient convenience and a lower overall cost than other regimens. However, because of the limited data available, further clinical research with capecitabine is still necessary to establish the optimum strategy.

\section{Future perspective}

In China, capecitabine-based chemotherapy regimens have been shown to offer survival benefits. The challenge for the physicians is how and where to place additional treatment modalities (i.e., chemotherapy or CRT; adjuvant or other settings). The selection of appropriate patients may achieve better outcomes. In the next 5-10 years, more data collected from high-quality studies in China will offer an optimal strategy in individualize treatment.

\section{Financial \& competing interests disclosure}

This review article is sponsored by Shanghai Roche Pharmaceutical Company. Y Lu is an employee of Roche Pharmaceutical Company. The authors have no other relevant affiliations or financial involvement with any organization or entity with a financial interest in or financial conflict with the subject matter or materials discussed in the manuscript apart from those disclosed.

Medical writing services were provided by $Y$ Yang of Content Ed Net Shanghai Company, under the guidance of two authors. The medical writing was paid for by Shanghai Roche Pharmaceutical Company.

\section{Open access}

This work is licensed under the Attribution-NonCommercial-NoDerivatives 4.0 Unported License. To view a copy of this license, visit http://creativecommons.org/licenses/by-nc-nd/4.0/ 
- In China, the treatment of locally advanced gastric cancer (AGC) faces unique challenges, as the Chinese population may harbor more unfavorable factors associated with poor survival than other populations, and a higher proportion of Chinese patients are diagnosed with AGC due to inadequate early diagnosis of the malignancy.

- Consequently, the optimal adjuvant chemotherapy strategy and clinical decision-making steps for Chinese patients cannot easily be duplicated from other countries because of the unique characteristics of gastric cancer in the Chinese population.

Capecitabine-based regimens for adjuvant chemotherapy of AGC

- Consistent with studies in other countries, capecitabine-based adjuvant chemotherapy regimens have been found to offer a significant survival benefit and a tolerable safety profile for postoperative AGC patients in China.

- From a review of the available clinical data in Chinese patients, certain treatment options may achieve better patient outcomes under specific conditions:

- XELOX (capecitabine + oxaliplatin), which has been the most commonly used adjuvant regimen in China, appears suitable for the general AGC population;

- Capecitabine-based concurrent chemoradiotherapy, which has stronger antitumor activity but more severe toxicity, may be a suitable option for high-risk patients with later-stage or node-positive disease, or where suboptimal surgery (less than D2 dissection) has been performed;

- Low-dose capecitabine monotherapy may be a suitable option for elderly patients with AGC;

- Capecitabine maintenance therapy, which has proved well tolerated and efficient in reducing recurrences, may be an option for patients with a high risk of relapse;

- Intraperitoneal regimens, which have a positive effect in decreasing ascitic fluid and preventing peritoneal recurrence and distant metastasis (especially liver metastasis), may be considered for patients with pyoperitoneum, ascites and a high risk of metastasis.

Conclusion

- Capecitabine-based chemotherapy regimens, especially XELOX, offer good efficacy following radical gastrectomy in Chinese patients with AGC, with a low incidence of adverse events, acceptable tolerance, greater patient convenience and a lower overall cost than other regimens.

- However, because of the limited data available, further clinical research with capecitabine is still necessary to establish the optimum strategy.

\section{References}

Papers of special note have been highlighted as: $\bullet$ of interest; $\bullet \bullet$ of considerable interest

1 Ferlay J, Soerjomataram I, Dikshit R et al. Cancer incidence and mortality worldwide: sources, methods and major patterns in GLOBOCAN 2012. Int. J. Cancer 136(5), e359-e386 (2015).

2 Chen W, Zheng R, Baade PD et al. Cancer statistics in China, 2015. CA Cancer J. Clin. 66(2), 115-132 (2016).

3 Allemani C, Weir HK, Carreira H et al. Global surveillance of cancer survival 1995-2009: analysis of individual data for 25,676,887 patients from 279 population-based registries in 67 countries (CONCORD-2). Lancet 385(9972), 977-1010 (2015).

4 Strong VE, Wu AW, Selby LV et al. Differences in gastric cancer survival between the US and China. J. Surg. Oncol. 112(1), 31-37 (2015).

5 Smyth EC, Verheij M, Allum W et al. Gastric cancer: ESMO Clinical Practice Guidelines for diagnosis, treatment and follow-up. Ann. Oncol. 27(Suppl. 5), V38-V49 (2016).

6 Sasaki E, Maeda Y, Sasaki T. [Comparison the standard therapies of gastric cancer in Japan with those in the west]. Gan To Kagaku Ryoho 34(5), 700-704 (2007).

7 Yang K, Hu JK. Gastric cancer treatment: similarity and difference between China and Korea. Transl. Gastroenterol. Hepatol. 2, 36 (2017).

8 Noh SH, Park SR, Yang HK et al. Adjuvant capecitabine plus oxaliplatin for gastric cancer after D2 gastrectomy (CLASSIC): 5-year follow-up of an open-label, randomised Phase III trial. Lancet Oncol. 15(12), 1389-1396 (2014).

- A large multicenter Phase III trial.

9 NCCN guidelines for gastric cancer. Version 1 (2017). www.nccn.org/

10 NCCN guidelines for gastric cancer. Chinese edition (2011). www.nccn.org/

11 Ji J, Liang H, Zhan Y et al. [Adjuvant capecitabine and oxaliplatin for gastric cancer after D2 gastrectomy (CLASSIC): Chinese subgroup analysis]. Zhonghua Wei Chang Wai Ke Za Zhi (Chinese J. Gastrointestinal Surg.) 17(2), 133-138 (2014).

- A large Phase III trial conducted in China.

12 Zhou YM. The efficacy of adjuvant chemotherapy of oxaliplatin plus capecitabine in stage II-III gastric cancer patients. Strait Pharm. J. 7, 110-112 (2015). 
13 Zhou YM. The efficacy of adjuvant chemotherapy of oxaliplatin plus capecitabine in stage II-III gastric cancer patients. Strait Pharm. J. 7, 110-112 (2015).

14 Wu Y, Wei ZW, He YL et al. Efficacy of adjuvant XELOX and FOLFOX6 chemotherapy after D2 dissection for gastric cancer. World J. Gastroenterol. 19(21), 3309-3315 (2013).

15 Lei Z, Li H. Clinical observation of combination of oxaliplatin and capecitabine or oxaliplatin and 5-fluorouracil/leucovorin chemotherapy for local advanced gastric cancer. World Health Digest Medical Periodical 9(22), 40-42 (2012).

16 Chen S, Feng X, Li Y, Yuan X, Zhou Z, Chen Y. Efficacy and safety of XELOX and FOLFOX6 adjuvant chemotherapy following radical total gastrectomy. Oncol. Lett. 3(4), 781-786 (2012).

17 Feng J, Qin HF, Wang BL et al. Value of oxaliplatin-based program in gastric cancer chemotherapy. Chin. J. Dig. Dis. 33(4), 226-230 (2013).

18 Zhang ZD, Kong Y, Ma F et al. Adjuvant chemotherapy with oxaliplatin plus S-1 versus XELOX regimen for postoperative gastric cancer. Chin. J. Gen. Surg. 22(6), 747-751 (2013).

19 Li YF, Zhou ZW, Li W et al. Comparison of capecitabine plus lobaplatin or oxaliplatin as adjuvant chemocherapy in advanced gastric cancer after D2 resection. Guangdong Medical Journal 33(9), 1324-1327 (2012).

20 MacDonald JS, Smalley SR, Benedetti J et al. Chemoradiotherapy after surgery compared with surgery alone for adenocarcinoma of the stomach or gastroesophageal junction. N. Engl. J. Med. 345(10), 725-730 (2001).

-. A USA hallmark study.

21 Dikken JL, van Sandick JW, Maurits Swellengrebel HA et al. Neo-adjuvant chemotherapy followed by surgery and chemotherapy or by surgery and chemoradiotherapy for patients with resectable gastric cancer (CRITICS). BMC Cancer 11, 329 (2011).

- A European hallmark study.

22 Velenik V, Anderluh F, Oblak I, Strojan P, Zakotnik B. Capecitabine as a radiosensitizing agent in neoadjuvant treatment of locally advanced resectable rectal cancer: prospective Phase II trial. Croat. Med. J. 47(5), 693-700 (2006).

23 Qiu H, Ding FY, Xiong HH, Li RC. A contrast research of concurrent chemoradiotherapy by capecitabine and 5-fluorouracil for gastric cancer. Herald of Medicine 29(3), 306-309 (2010).

24 Shi XG. The observation of capecitabine based concurrent chemoradiotherapy after radical gastrectomy. Journal of Clinical Medical 3(17), 3477-3477 (2016)

- Largest randomized, controlled trial of capecitabine-based chemoradiotherapy in China.

25 Chen BG, Cai YC, Mo RK, Liu TQ, Li JX. Clinical study of three dimensional conformal radiation therapy plus capecitabine chemotherapy after radical gastrectomy. International Medicine \& Health Guidance News 18(7), 942-945 (2012).

26 Guo P, Chen JF, Huang W et al. Observation on efficacy of docetaxel combined with capecitabine chemotherapy and concurrent radiochemotherapy in advanced gastric cancer after surgery. Evaluation and Analysis of Drug Use in Hospitals of China 16(8), 1055-1057 (2016).

27 Zhang YS, Zhao CH, Wang YM et al. Efficacy analysis of capecitabine based concurrent radiochemotherapy in 50 gastric cancer patients after surgery. China Pharmaceuticals 5, 65-67 (2015).

28 Yuan ST, Wang FL, Liu N et al. Concurrent involved-field radiotherapy and XELOX versus XELOX chemotherapy alone in gastric cancer patients with postoperative locoregional recurrence. Am. J. Clin. Oncol. 38(2), 130-134 (2015).

29 Luo W, Zhang H, Zhao Y et al. A retrospective study on intensity-modulated radiation therapy combined with chemotherapy after D2 radical surgery for gastric carcinoma. Mol. Clin. Oncol. 4(5), 740-748 (2016).

30 Ren $\mathrm{H}$, Wang X, Fang $\mathrm{H}$ et al. Interim assessment of prospective Phase II trial evaluating efficacy of intensity-modulated radiotherapy with concurrent capecitabine for stage II/III gastric cancer after radical surgery. Clin. J. Radiat. Oncol. 23(2), 104-107 (2014).

31 Wang YW, Tang YW. A preliminary study on toxicity and efficacy of post-operative 3D radiotherapy plus capecitabine in treatment of patients with advanced gastric cancer. J. Clin. Exp. Med. 11(16), 1283-1284 (2012).

32 Wang X, Jin J, Li YX et al. Phase I trial of postoperative concurrent capecitabine and intensity-modulated radiotherapy for locally advanced gastric cancer. Chin. J. Radiat. Oncol. 22(5), 343-346 (2013).

33 Wang X, Jin J, Li YX et al. Phase I study of postoperative radiotherapy combined with capecitabine for gastric cancer. World J. Gastroenterol. 20(4), 1067-1073 (2014).

34 Bickenbach K, Strong VE. Comparisons of gastric cancer treatments: east vs west. J. Gastric Cancer 12(2), 55-62 (2012).

35 Lee J, Lim DH, Kim S et al. Phase III trial comparing capecitabine plus cisplatin versus capecitabine plus cisplatin with concurrent capecitabine radiotherapy in completely resected gastric cancer with D2 lymph node dissection: the ARTIST trial. J. Clin. Oncol. 30(3), 268-273 (2012).

36 Foo M, Leong T. Adjuvant therapy for gastric cancer: current and future directions. World J. Gastroenterol. 20(38), 13718-13727 (2014).

37 Li L, Zhang JH. Clinical efficacy of capecitabine treatment in elderly patients with advanced gastric cancer. Anti-tumor Pharmacy 5 , 379-380 (2012). 
38 Yuan X. Clinical application of capecitabine as adjuvant chemotherapy of gastric cancer in aged patients. Clinical Medicine of China 25(7), 750-752 (2009).

39 Bajetta E, Procopio G, Celio L et al. Safety and efficacy of two different doses of capecitabine in the treatment of advanced breast cancer in older women. J. Clin. Oncol. 23(10), 2155-2161 (2005).

40 Feliu J, Escudero P, Llosa F et al. Capecitabine as first-line treatment for patients older than 70 years with metastatic colorectal cancer: an Oncopaz Cooperative Group study. J. Clin. Oncol. 23(13), 3104-3111 (2005).

41 Coccolini F, Cotte E, Glehen $\mathrm{O}$ et al. Intraperitoneal chemotherapy in advanced gastric cancer: meta-analysis of randomized trials. Eur. J. Surg. Oncol. 40(1), 12-26 (2014).

- Meta-analysis of intraperitoneal chemotherapy in advanced gastric cancer.

42 Zhong L, Cai MD, Wei HJ, Zeng H, Li JF. Clinical observation of intraperitoneal cisplatin plus oral capecitabine therapy in advanced gastric cancer patients after surgery. Youjiang Medical Journal 40(3), 341-342 (2012).

43 Wang SC, Bai W. Clinical observation of intraperitoneal docetaxel and cisplatin plus oral capecitabine therapy in gastric cancer patients with pyoperitoneum. Cancer Research and Clinic 27(1), 55-56 (2015).

44 Feng WM, Tang CW, Guo HH, Bao Y, Fei MY. Prolonged adjuvant capecitabine chemotherapy improved survival of stage IIIa gastric cancer after D2 gastrectomy. Biomed. Pharmacother. 72, 140-143 (2015).

$45 \mathrm{Qu}$ J, Ren XA, Bian JL, Tang SH. Capecitabine used for maintenance therapy of adjuvant chemotherapy after operation of late gastric cancer patients. China Medicine and Pharmacy 1(9), 110 (2011).

46 Cassidy J, Saltz L, Twelves C et al. Efficacy of capecitabine versus 5-fluorouracil in colorectal and gastric cancers: a meta-analysis of individual data from 6171 patients. Ann. Oncol. 22(12), 2604-2609 (2011).

47 Cassidy J, Twelves C, Van Cutsem E et al. First-line oral capecitabine therapy in metastatic colorectal cancer: a favorable safety profile compared with intravenous 5-fluorouracil/leucovorin. Ann. Oncol. 13(4), 566-575 (2002).

48 Hoff PM, Ansari R, Batist G et al. Comparison of oral capecitabine versus intravenous fluorouracil plus leucovorin as first-line treatment in 605 patients with metastatic colorectal cancer: results of a randomized Phase III study. J. Clin. Oncol. 19(8), 2282-2292 (2001).

49 Twelves C, Gollins S, Grieve R, Samuel L. A randomised cross-over trial comparing patient preference for oral capecitabine and 5-fluorouracil/leucovorin regimens in patients with advanced colorectal cancer. Ann. Oncol. 17(2), 239-245 (2006).

50 Chen YP, Hu SL. Analysis of the medical cost of capecitabine in treatment of advanced rectocolonic carcinoma. China Pharmacy 15(7), 415-416 (2004).

- Pharmacoeconomic considerations relating to capecitabine.

51 Wang L, Liu ZH. Pharmacoeconomic evaluation of selective targeted oral chemotherapeutic agent Xeloda. Health Vocational Education 24(3), 154-157 (2006).

52 Wu B, Li T, Cai J, Xu Y, Zhao G. Cost-effectiveness analysis of adjuvant chemotherapies in patients presenting with gastric cancer after D2 gastrectomy. BMC Cancer 14, 984 (2014).

53 Sasako M, Sakuramoto S, Katai H et al. Five-year outcomes of a randomized Phase III trial comparing adjuvant chemotherapy with S-1 versus surgery alone in stage II or III gastric cancer. J. Clin. Oncol. 29(33), 4387-4393 (2011).

54 Li Z, Fan B, Shan F et al. Gastrectomy in comprehensive treatment of advanced gastric cancer with synchronous liver metastasis: a prospectively comparative study. World J. Surg. Oncol. 13, 212 (2015). 
